\title{
EXISTENCE AND UNIQUENESS IN THE THEORY OF BENDING OF ELASTIC PLATES
}

\author{
by CHRISTIAN CONSTANDA
}

(Received 19th November 1984)

\section{Introduction}

Kirchhoff's kinematic hypothesis that leads to an approximate two-dimensional theory of bending of elastic plates consists in assuming that the displacements have the form [1]

$$
\begin{aligned}
& u_{\alpha}=x_{3} v_{\alpha}\left(x_{1}, x_{2}\right), \quad \alpha=1,2, \\
& u_{3}=v_{3}\left(x_{1}, x_{2}\right)
\end{aligned}
$$

In general, the Dirichlet and Neumann problems for the equilibrium equations obtained on the basis of (1.1) cannot be solved by the boundary integral equation method both inside and outside a bounded domain because the corresponding matrix of fundamental solutions does not vanish at infinity [2]. However, as we show in this paper, the method is still applicable if the asymptotic behaviour of the solution is suitably restricted.

\section{Notation and formulae}

Let $\left(x_{1}, x_{2}, x_{3}\right)$ be the Cartesian coordinates of a generic point in $R^{3}$. We consider a homogeneous and isotropic plate occupying a region $\Omega \times\left[-h_{0} / 2, h_{0} / 2\right]$, where $h_{0}=$ const and $\Omega$ is a domain in the $\left(x_{1}, x_{2}\right)$-plane, whose boundary $\partial \Omega$ is a closed Lyapunov curve.

Unless otherwise stipulated, throughout the paper Latin and Greek suffixes take the values $1,2,3$ and 1,2 , respectively, the convention of summation over repeated indices is adopted, and $(\ldots)_{\alpha}=\partial(\ldots) / \partial x_{\alpha}$.

Also, we denote by $M^{*}$ the transpose of a matrix $M$, and by $H_{k}$ and $J_{k}(k=0,1)$ the averaging operators

$$
\begin{aligned}
& H_{k} w\left(x_{i}\right)=\left(1 / h_{0}\right)\left[x_{3}^{k} w\left(x_{i}\right)\right]_{x_{3}=-h_{0} / 2}^{x_{3}=h_{0} / 2}, \\
& J_{k} w\left(x_{i}\right)=\left(1 / h_{0}\right) \int_{-h_{0} / 2}^{h_{0} / 2} x_{3}^{k} w\left(x_{i}\right) d x_{3} .
\end{aligned}
$$

Let $t_{i j}$ be the stresses, $u_{i}$ the displacements, $f_{i}$ the body forces, and $\lambda$ and $\mu$ the elastic coefficients of the material. If we set

$$
F_{\alpha}=-\left(J_{1} f_{\alpha}+H_{1} t_{3 \alpha}\right), \quad F_{3}=-\left(J_{0} f_{3}+H_{0} t_{33}\right)
$$


then the linear equilibrium equations obtained by using (1.1) can be written in the form

$$
L\left(\partial_{x}\right) v(x)=F(x)
$$

where $L\left(\partial_{x}\right)=L\left(\partial / \partial x_{1}, \partial / \partial x_{2}\right)=\left(L_{i j}\left(\partial_{x}\right)\right)$

$$
L\left(\xi_{1}, \xi_{2}\right)=\left(\begin{array}{ccc}
h^{2} \mu \Delta+h^{2}(\lambda+\mu) \xi_{1}^{2}-\mu & h^{2}(\lambda+\mu) \xi_{1} \xi_{2} & -\mu \xi_{1} \\
h^{2}(\lambda+\mu) \xi_{1} \xi_{2} & h^{2} \mu \Delta+h^{2}(\lambda+\mu) \xi_{2}^{2}-\mu & -\mu \xi_{2} \\
\mu \xi_{1} & \mu \xi_{2} & \mu \Delta
\end{array}\right)
$$

$v^{*}=\left(v_{1}, v_{2}, v_{3}\right), F^{*}=\left(F_{1}, F_{2}, F_{3}\right), \Delta=\xi_{\alpha} \xi_{\alpha}$, and $h^{2}=h_{0}^{2} / 12$.

In connection with the system (2.1) we consider the boundary stress operator $T\left(\partial_{x}\right)=$ $T\left(\partial / \partial x_{1}, \partial / \partial x_{2}\right)=\left(T_{i j}\left(\partial_{x}\right)\right)$, where

$$
T\left(\xi_{1}, \xi_{2}\right)=\left(\begin{array}{ccc}
h^{2}(\lambda+2 \mu) n_{1} \xi_{1}+h^{2} \mu n_{2} \xi_{2} & h^{2} \mu n_{2} \xi_{1}+h^{2} \lambda n_{1} \xi_{2} & 0 \\
h^{2} \lambda n_{2} \xi_{1}+h^{2} \mu n_{1} \xi_{2} & h^{2} \mu n_{1} \xi_{1}+h^{2}(\lambda+2 \mu) n_{2} \xi_{2} & 0 \\
\mu n_{1} & \mu n_{2} & \mu\left(n_{1} \xi_{1}+n_{2} \xi_{2}\right)
\end{array}\right)
$$

and $n_{\alpha}$ are the components of the unit outward normal to $\partial \Omega$.

In what follows we assume that $\lambda$ and $\mu$ satisfy the inequalities

$$
\lambda+\mu>0, \quad \mu>0,
$$

which ensure that the system (2.1) is elliptic and that the internal energy of the plate (measured on the unit of its middle plane) $h_{0} E(v, v)$, where

$$
2 E(v, v)=h^{2}\left[\lambda v_{\alpha, \alpha} v_{\beta, \beta}+\mu\left(v_{\alpha, \beta}+v_{\beta, \alpha}\right) v_{\alpha, \beta}\right]+\mu\left(v_{3, \alpha}+v_{\alpha}\right)\left(v_{3, \alpha}+v_{\alpha}\right),
$$

is positive.

From (2.1)-(2.4) we easily obtain the Betti formula

$$
\int_{\mathbf{\Omega}} v^{*} L v d \sigma+\int_{\Omega} 2 E(v, v) d \sigma=\int_{\partial \Omega} v^{*} T v d s
$$

and the relations

$$
\begin{gathered}
\int_{\Omega} L_{3 i} v_{i} d \sigma=\int_{\partial \Omega} T_{3 i} v_{i} d s \\
\left.\int_{\Omega} L_{\alpha i}-x_{\alpha} L_{3 i}\right) v_{i} d \sigma=\int_{\partial \Omega}\left(T_{\alpha i}-x_{\alpha} T_{3 i}\right) v_{i} d s
\end{gathered}
$$

where $v$ is a smooth $(3 \times 1)$-matrix.

\section{Fundamental solutions}

The matrix of fundamental solutions for the system (2.1) is [2]

$$
D(x, y)=B\left(\partial_{x}\right) t(x, y)
$$


where

$$
\begin{gathered}
t(x, y)=t(r)=a\left[r^{2} \ln r+4 h^{2} \ln r+4 h^{2} K_{0}(r / h)\right], \\
a=\left[8 \pi h^{2} \mu^{2}(\lambda+2 \mu)\right]^{-1} \\
r=|x-y|=\left[\left(x_{1}-y_{1}\right)^{2}+\left(x_{2}-y_{2}\right)^{2}\right]^{1 / 2}
\end{gathered}
$$

$K_{0}(\xi)$ is the modified Bessel function of order zero, and the entries of the matrix $B\left(\partial_{x}\right)=$ $\left(B_{i j}\left(\partial / \partial x_{1}, \partial / \partial x_{2}\right)\right)$ are

$$
\begin{aligned}
& B_{\alpha \alpha}(\xi)=h^{2} \mu(\lambda+2 \mu) \Delta^{2}-h^{2} \mu(\lambda+\mu) \Delta \xi_{\alpha}^{2}-\mu^{2} \xi_{\alpha}^{2}, \quad(\alpha \text { not summed }) \\
& B_{33}(\xi)=h^{4} \mu(\lambda+2 \mu) \Delta^{2}-h^{2} \mu(\lambda+3 \mu) \Delta+\mu^{2} \\
& B_{12}(\xi)=B_{21}(\xi)=-\mu \xi_{1} \xi_{2}\left[h^{2}(\lambda+\mu) \Delta+\mu\right] \\
& B_{\alpha 3}(\xi)=-B_{3 \alpha}(\xi)=\mu^{2} \xi_{\alpha}\left(h^{2} \Delta-1\right) .
\end{aligned}
$$

We also consider the matrix $P(x, y)=\left(P_{i j}(x, y)\right)$, where

$$
P_{i j}(x, y)=\left(T\left(\partial_{y}\right) D(y, x)\right)_{j i}
$$

We denote $\Omega$ by $\Omega_{\text {in }}$ and set $\Omega_{\mathrm{ex}}=R^{2} \backslash\left\{\Omega_{\mathrm{in}} \cup \partial \Omega\right\}$. As in the classical theory (see, for example, [3]), we can derive the relations

$$
\begin{gathered}
\int_{\partial \Omega} T_{i k}\left(\partial_{x}\right) D_{k j}(x, y) d s_{x}=\phi(y) \delta_{i j} \\
\int_{\partial \Omega} x_{\alpha} T_{3 k}\left(\partial_{x}\right) D_{k j}(x, y) d s_{x}=y_{\alpha} \phi(y) \delta_{3 j}
\end{gathered}
$$

where

$$
\phi(y)= \begin{cases}-1, & y \in \Omega_{\mathrm{in}} \\ -1 / 2, & y \in \partial \Omega \\ 0, & y \in \Omega_{\mathrm{ex}}\end{cases}
$$

and $\delta_{i j}$ are the Kronecker delta, and the Somigliana formula

$$
\begin{aligned}
v(x)= & \int_{\partial \Omega}\left[D(x, y) T\left(\partial_{y}\right) v(y)-P(x, y) v(y)\right] d s_{y} \\
& -\int_{\Omega_{\mathrm{in}}} D(x, y) L\left(\partial_{y}\right) v(y) d s_{y}, \quad x \in \Omega_{\mathrm{in}},
\end{aligned}
$$

where $v$ is a smooth $(3 \times 1)$-matrix.

Let $\mathscr{A}$ be the set of $(3 \times 1)$-matrices $v$ in $\Omega_{\mathrm{ex}}$ having an asymptotic expansion of the 
form

$$
\begin{aligned}
v_{1}(r, \theta)= & \frac{1}{r}\left[\alpha_{0} \sin \theta+2 \alpha_{1} \cos \theta-\alpha_{0} \sin 3 \theta+\left(\alpha_{2}-\alpha_{1}\right) \cos 3 \theta\right] \\
& +\frac{1}{r^{2}}\left[\left(2 \beta_{1}+\gamma_{1}\right) \sin 2 \theta+\gamma_{2} \cos 2 \theta-2 \beta_{1} \sin 4 \theta+2 \beta_{2} \cos 4 \theta\right] \\
& +\frac{1}{r^{3}}\left[2 \rho_{1} \sin 3 \theta+2 \pi_{1} \cos 3 \theta+3\left(\rho_{2}-\rho_{1}\right) \sin 5 \theta+3\left(\pi_{2}-\pi_{1}\right) \cos 5 \theta\right]+O\left(\frac{1}{r^{4}}\right), \\
v_{2}(r, \theta)= & \frac{1}{r}\left[2 \alpha_{2} \sin \theta+\alpha_{0} \cos \theta+\left(\alpha_{2}-\alpha_{1}\right) \sin 3 \theta+\alpha_{0} \cos 3 \theta\right] \\
& +\frac{1}{r^{2}}\left[\left(2 \beta_{2}+\gamma_{2}\right) \sin 2 \theta-\gamma_{1} \cos 2 \theta+2 \beta_{2} \sin 4 \theta+2 \beta_{1} \cos 4 \theta\right] \\
& +\frac{1}{r^{3}}\left[2 \pi_{2} \sin 3 \theta-2 \rho_{2} \cos 3 \theta+3\left(\pi_{2}-\pi_{1}\right) \sin 5 \theta+3\left(\rho_{1}-\rho_{2}\right) \cos 5 \theta\right]+O\left(\frac{1}{r^{4}}\right), \\
& +\frac{1}{r^{2}}\left[\sigma_{1} \sin 2 \theta+\sigma_{2} \cos 2 \theta+\left(\rho_{2}-\rho_{1}\right) \sin 4 \theta+\left(\pi_{2}-\pi_{1}\right) \cos 4 \theta\right]+O\left(\frac{1}{r^{3}}\right), \\
& +\frac{1}{r}\left[\left(\beta_{1}+\gamma_{1}\right) \sin \theta+\left(\beta_{2}+\gamma_{2}\right) \cos \theta-\beta_{1} \sin 3 \theta+\beta_{2} \cos 3 \theta\right] \\
v_{3}(r, \theta)= & \left(\alpha_{1}+\alpha_{2}\right) \ln r-\left[\alpha_{1}+\alpha_{2}+\alpha_{0} \sin 2 \theta+\left(\alpha_{1}-\alpha_{2}\right) \cos 2 \theta\right] \\
& \\
& \\
&
\end{aligned}
$$

where $\alpha_{0}, \alpha_{v}, \beta_{v}, \gamma_{v}, \rho_{v}, \pi_{v}, \sigma_{v}=$ const, and $\mathscr{A}^{\prime}$ the set of matrices $v^{\prime}=v+C$, where $v \in \mathscr{A}$ and

$$
C^{*}=\left(c_{1}, c_{2}, c-c_{1} x_{1}-c_{2} x_{2}\right), \quad c, c_{\alpha}=\text { const }
$$

Remark. In view of (1.1), the matrix (3.7) represents a rigid displacement, and it is obvious that for $v \in \mathscr{A}^{\prime}$ the internal energy density (2.6) remains finite as $r \rightarrow \infty$.

\section{Elastic potentials}

We define the single layer potential

$$
V(x)=\int_{\partial \Omega} D(x, y) z(y) d s_{y}
$$

and the double layer potential

$$
W(x)=\int_{\partial \Omega} P(x, y) z(y) d s_{y}
$$


where the $(3 \times 1)$-matrix $z$ is an unknown density. Proceeding as in [4], we can prove the following assertions:

Theorem 1. $L\left(\partial_{x}\right) V(x)=0, L\left(\partial_{x}\right) W(x)=0, x \notin \partial \Omega$.

Theorem 2. (i) If $z(x)$ is continuous on $\partial \Omega$, then $V(x)$ is continuous in $R^{2}$.

(ii) If $z(x)$ is Hölder continuous on $\partial \Omega$, then $W(x)$ tends to finite limits as $x \rightarrow x_{0} \in \partial \Omega$ both from $\Omega_{\mathrm{in}}$ and from $\Omega_{\mathrm{ex}}$, these limits being

$$
\begin{gathered}
W_{\mathrm{in}}\left(x_{0}\right)=-\frac{1}{2} z\left(x_{0}\right)+\int_{\partial \Omega} P\left(x_{0}, y\right) z(y) d s_{y} \\
W_{\mathrm{ex}}\left(x_{0}\right)=\frac{1}{2} z\left(x_{0}\right)+\int_{\partial \Omega} P\left(x_{0}, y\right) z(y) d s_{y}
\end{gathered}
$$

(iii) If $z(x)$ is Hölder continuous on $\partial \Omega$, then $T\left(\partial_{x}\right) V(x)$ tends to finite limits as $x \rightarrow x_{0} \in \partial \Omega$ both from $\Omega_{\mathrm{in}}$ and from $\Omega_{\mathrm{ex}}$, these limits being

$$
\begin{gathered}
(T V)_{\mathrm{in}}\left(x_{0}\right)=\frac{1}{2} z\left(x_{0}\right)+\int_{\partial \Omega} T\left(\partial_{x_{0}}\right) D\left(x_{0}, y\right) z(y) d s_{y}, \\
(T V)_{\mathrm{ex}}\left(x_{0}\right)=-\frac{1}{2} z\left(x_{0}\right)+\int_{\partial \Omega} T\left(\partial_{x_{0}}\right) D\left(x_{0}, y\right) z(y) d s_{y} .
\end{gathered}
$$

Also, we have [2]

Theorem 3. If $z(x)$ is continuous on $\partial \Omega$, then

(i) $W \in \mathscr{A}$;

(ii) $V \in \mathscr{A}$ if and only if

$$
\int_{\partial \Omega} z_{3} d s=\int_{\partial \Omega}\left(z_{\alpha}-x_{\alpha} z_{3}\right) d s=0
$$

\section{Boundary value problems}

Let $A(x), B(x), R(x)$, and $S(x)$ be $(3 \times 1)$-matrices prescribed on $\partial \Omega$. For the homogeneous system (2.1), that is,

$$
L\left(\partial_{x}\right) v(x)=0
$$

we consider the following Dirichlet and Neumann problems:

(I) Find $v$ satisfying $(5.1)$ in $\Omega_{\text {in }}$ and

$$
v(x)=A(x), \quad x \in \partial \Omega
$$


(II) Find $v$ satisfying (5.1) in $\Omega_{\text {in }}$ and

$$
T\left(\partial_{x}\right) v(x)=B(x), \quad x \in \partial \Omega
$$

(III) Find $v \in \mathscr{A}^{\prime}$ satisfying (5.1) in $\Omega_{\mathrm{ex}}$ and

$$
v(x)=R(x), \quad x \in \partial \Omega .
$$

(IV) Find $v \in \mathscr{A}$ satisfying (5.1) in $\Omega_{\mathrm{ex}}$ and

$$
T\left(\partial_{x}\right) v(x)=S(x), \quad x \in \partial \Omega
$$

Theorem 4. (i) The interior Dirichlet problem (I) has a unique solution for any Hölder continuous matrix $A(x)$. This solution can be expressed as a double layer potential.

(ii) The exterior Neumann problem (IV) has a unique solution for any Hölder continuous matrix $S(x)$ if and only if

$$
\int_{\partial \Omega} S_{3} d s=\int_{\partial \Omega}\left(S_{\alpha}-x_{\alpha} S_{3}\right) d s=0
$$

The solution can be expressed as a single layer potential.

Proof. First, suppose that $v \in \mathscr{A}$ is a solution of (IV). Let $K_{R}$ be a circle whose radius $R$ is sufficiently large so that $(\Omega \cup \partial \Omega) \subset K_{R}$. Applying (2.6) to $v$ in $\Omega_{\mathrm{ex}} \cap K_{R}$, we arrive at the equalities

$$
\begin{aligned}
& \int_{\partial \Omega} S_{3} d s=\int_{\partial \Omega} T_{3 i} v_{i} d s=-\int_{\Omega_{e x} \cap K_{R}} L_{3 i} v_{i} d \sigma+\int_{\partial K_{R}} T_{3 i} v_{i} d s=\int_{\partial K_{R}} T_{3 i} v_{i} d s, \\
& \int_{\partial \Omega}\left(S_{\alpha}-x_{\alpha} S_{3}\right) d s=\int_{\partial \Omega}\left(T_{\alpha i}-x_{\alpha} T_{3 i}\right) v_{i} d s \\
& =-\int_{\Omega_{\mathrm{ex}} \cap K_{R}}\left(L_{\alpha i}-x_{\alpha} L_{3 i}\right) v_{i} d \sigma+\int_{\partial K_{R}}\left(T_{\alpha i}-x_{\alpha} T_{3 i}\right) v_{i} d s .
\end{aligned}
$$

Since $T_{\alpha i} v_{i}=O\left(1 / R^{2}\right)$ and $T_{3 i} v_{i}=O\left(1 / R^{3}\right)$ for $v \in \mathscr{A}$ [2], the relations (5.6) are obtained immediately by letting $R \rightarrow \infty$.

Suppose now that (5.6) hold. Seeking the solutions of (I) and (IV) in the form of $W$ and $V$, respectively, we interpret (5.2) and (5.5) as

$$
\begin{gathered}
W_{\text {in }}(x)=A(x), \quad x \in \partial \Omega, \\
(T V)_{e x}(x)=S(x), \quad x \in \partial \Omega,
\end{gathered}
$$

and from Theorem 2(ii, iii) it follows that the unknown density $z(x)$ must satisfy the 
integral equation

$$
-\frac{1}{2} z(x)+\int_{\partial \Omega} P(x, y) z(y) d s_{y}=A(x), \quad x \in \partial \Omega
$$

(in the case of $(\mathrm{I})$ ), and

$$
-\frac{1}{2} z(x)+\int_{\partial \Omega} T\left(\partial_{x}\right) D(x, y) z(y) d s_{y}=S(x), \quad x \in \partial \Omega
$$

(in the case of (IV)).

In view of (3.4), $\left(D_{\mathrm{in}}\right)$ and $\left(N_{\mathrm{ex}}\right)$ are mutually adjoint, and using (3.1)-(3.4) it is not difficult to show that their index [5] is zero, which implies that Fredholm's theorems are applicable to these equations.

Multiplying $\left(N_{\mathrm{ex}}\right)_{3}$ and the combination $\left(N_{\mathrm{ex}}\right)_{\alpha}-x_{\alpha} \times\left(N_{\mathrm{ex}}\right)_{3}$ by $d s_{x}$, integrating over $\partial \Omega$, and taking (3.5) into account, we obtain

$$
\int_{\partial \Omega} z_{3} d s=-\int_{\partial \Omega} S_{3} d s, \quad \int_{\partial \Omega}\left(z_{\alpha}-x_{\alpha} z_{3}\right) d s=-\int_{\partial \Omega}\left(S_{\alpha}-x_{\alpha} S_{3}\right) d s .
$$

Suppose the existence of a non-trivial solution $z^{0}(x)$ to the homogeneous equation $\left(N_{\mathrm{ex}}^{0}\right)$, that is,

$$
-\frac{1}{2} z^{0}(x)+\int_{\partial \Omega} T\left(\partial_{x}\right) D(x, y) z^{0}(y) d s_{y}=0, \quad x \in \partial \Omega
$$

Then, by Theorem 2(iii), the single layer potential

$$
V^{0}(x)=\int_{\partial \Omega} D(x, y) z^{0}(y) d s_{y}
$$

satisfies

$$
\left(T V^{0}\right)_{e x}(x)=-\frac{1}{2} z^{0}(x)+\int_{\partial \Omega} T\left(\partial_{x}\right) D(x, y) z^{0}(y) d s_{y}=0, \quad x \in \partial \Omega
$$

and from (5.7) we find that

$$
\int_{\partial \Omega} z_{3}^{0} d s=\int_{\partial \Omega}\left(z_{\alpha}^{0}-x_{\alpha} z_{3}^{0}\right) d s=0
$$

By Theorem 3(ii), $V^{0} \in \mathscr{A}$. Since, according to Theorems 1 and 2(iii), we also have

$$
\begin{array}{ll}
L\left(\partial_{x}\right) V^{0}(x)=0, & x \in \Omega_{e x} \\
\left(T V^{0}\right)_{e x}(x)=0, & x \in \partial \Omega,
\end{array}
$$

the uniqueness theorem for (IV) [2] implies that $V^{0}(x) \equiv 0, x \in \Omega_{e x}$. Theorem 2(i) now 
yields $V^{0}(x)=0, x \in \partial \Omega$, and since $L\left(\partial_{x}\right) V^{0}(x)=0, x \in \Omega_{\mathrm{in}}$, from the uniqueness theorem for (I) we conclude that $V^{0}(x) \equiv 0, x \in R^{2}$. Finally, from (4.1) we obtain

$$
z^{\mathrm{o}}(x)=\left(T V^{0}\right)_{\mathrm{in}}(x)-\left(T V^{0}\right)_{\mathrm{ex}}(x)=0, \quad x \in \partial \Omega .
$$

This shows that $\left(N_{\mathrm{ex}}^{\mathbf{0}}\right)$ has only the trivial solution. Hence, so does the homogeneous equation $\left(D_{\mathrm{in}}^{0}\right)$, which means that both $\left(D_{\mathrm{in}}\right)$ and $\left(N_{\mathrm{ex}}\right)$ are uniquely solvable. To complete the proof we only need to check that in the case of (IV) the solution $V$ belongs to $\mathscr{A}$. But this is established immediately, since the relations (4.2) hold for the density $z(x)$ of $V$ by virtue of (5.6) and (5.7).

Theorem 5. The interior Neumann problem (II) is solvable for any Hölder continuous matrix $B(x)$ if and only if

$$
\int_{\partial \Omega} B_{3} d s=\int_{\partial \Omega}\left(B_{\alpha}-x_{\alpha} B_{3}\right) d s=0
$$

The solution can be expressed as a single layer potential and is unique up to a rigid displacement (3.7).

Proof. We seek the solution in the form of $V$ and interpret (5.3) as

$$
(T V)_{\text {in }}(x)=B(x), \quad x \in \partial \Omega
$$

By Theorem 2(iii), this means that $z(x)$ must satisfy the integral equation

$$
\frac{1}{2} z(x)+\int_{\partial \Omega} T\left(\partial_{x}\right) D(x, y) z(y) d s_{y}=B(x), \quad x \in \partial \Omega
$$

for which we can again verify that the index is zero.

It is easy to check that a matrix $C$ of the form (3.7) satisfies

$$
\begin{array}{ll}
L\left(\partial_{x}\right) C(x)=0, & x \in \Omega_{\mathrm{in}} \\
T\left(\partial_{x}\right) C(x)=0, & x \in \partial \Omega .
\end{array}
$$

Therefore, by (3.6), we have the representation

$$
C(x)=-\int_{\partial \Omega} P(x, y) C(y) d s_{y}, \quad x \in \Omega_{\mathrm{in}}
$$

Since the right-hand side above is a double layer potential, letting $x \rightarrow x_{0} \in \partial \Omega$ and making use of Theorem 2(ii) we obtain

$$
\frac{1}{2} C\left(x_{0}\right)+\int_{\partial \Omega} P\left(x_{0}, y\right) C(y) d s_{y}=0
$$


which means that $C$ is a solution of the adjoint homogeneous equation. Clearly, $v^{(i)}$, where

$$
v^{(1) *}=\left(1,0,-x_{1}\right), \quad v^{(2) *}=\left(0,1,-x_{2}\right), \quad v^{(3) *}=(0,0,1)
$$

are linearly independent solutions of the latter, so the homogeneous equation $\left(N_{\mathrm{in}}^{0}\right)$ has at least three linearly independent solutions $\mu^{(i)}$. Just as in [4], we can prove that $\left\{\mu^{(i)}\right\}$ is a complete system of such solutions for $\left(N_{\mathrm{in}}^{0}\right)$, hence, so is $\left\{v^{(i)}\right\}$ for (5.9). This implies that $\left(N_{\text {in }}\right)$ is solvable if and only if

$$
\int_{\partial \Omega} v^{(i) *} B d s=0
$$

which is equivalent to (5.8).

The last part of the statement of the theorem is obvious.

Remark. Taking into account (1.1) and the averaging procedure employed to derive the simplified two-dimensional bending equations, it can be shown that in the case of both Neumann problems (II) and (IV) the conditions (5.6) and (5.8) imply that the total force and moment across $\partial \Omega$ must be zero.

Theorem 6. The exterior Dirichlet problem (III) has a unique solution for any Hölder continuous matrix $R(x)$.

Proof. We assume that the systems of functions $\left\{\mu^{(i)}\right\}$ and $\left\{v^{(i)}\right\}$ mentioned in the proof of Theorem 5 have been bi-orthonormalized (by means of a procedure similar to that used in [4]) and seek the solution of (III) in the form $W+C_{i} v^{(i)}$, where $W$ is a double layer potential of unknown density $z$ and

$$
C_{i}=\int_{\partial \Omega} \mu^{(i) *} R d s
$$

Then Theorem 2(ii) yields

$$
\frac{1}{2} z(x)+\int_{\partial \Omega} P(x, y) z(y) d s_{y}=R(x)-C_{i} v^{(i)}(x), \quad x \in \partial \Omega
$$

In view of (3.4), this equation is adjoint to $\left(N_{\text {in }}\right)$, hence its index is zero. From the definition of the $C_{i}$ in (5.10) we see that

$$
\int_{\partial \Omega} \mu^{(k) *}\left(R-C_{i} v^{(i)}\right) d s=0
$$

which implies that $\left(D_{\mathrm{ex}}\right)$ is solvable. Also, by Theorem $3(\mathrm{i}), W \in \mathscr{A}$, and since $C_{i} v^{(i)}$ is a rigid displacement of the form (3.7), it follows that $W+C_{i} v^{(i)} \in \mathscr{A}^{\prime}$, as required. 


\section{REFERENCES}

1. P. M. NAGHDI, The theory of shells and plates (Handbuch der Physik VIa/2, Springer-Verlag, Berlin-Heidelberg-New York, 1978).

2. C. Constanda, Uniqueness in the theory of bending of elastic plates (to appear).

3. O. D. Kellog, Foundation of potential theory (Springer-Verlag, Berlin, 1929).

4. V. D. Kupradze, Potential methods in the theory of elasticity (Israel Program for Scientific Translations, Jerusalem, 1965).

5. N. I. MusKhelishviLI, Singular integral equations (P. Noordhoff, Groningen, 1951).

UNIVERSITY OF STRATHCLYDE

GLASGOW

SCOTLAND 